Homology, Homotopy and Applications, vol.22(1), 2020, pp.233-254

\title{
EROSION DISTANCE FOR GENERALIZED PERSISTENCE MODULES
}

\author{
VILLE PUUSKA
}

(communicated by Graham Ellis)

\begin{abstract}
The persistence diagram of Cohen-Steiner, Edelsbrunner, and Harer was recently generalized by Patel to the case of constructible persistence modules with values in a symmetric monoidal category with images. Patel also introduced a distance for persistence diagrams, the erosion distance. Motivated by this work, we extend the erosion distance to a distance of rank invariants of generalized persistence modules by using the generalization of the interleaving distance of Bubenik, de Silva, and Scott as a guideline. This extension of the erosion distance also gives, as a special case, a distance for multidimensional persistent homology groups with torsion introduced by Frosini. We show that the erosion distance is stable with respect to the interleaving distance, and that it gives a lower bound for the natural pseudo-distance in the case of sublevel set persistent homology of continuous functions.
\end{abstract}

\section{Introduction}

Persistent homology has risen to be a popular and powerful tool for extracting topological features of data sets (see [13] and [3]). Persistent homology takes a filtration of a topological space and computes the birth and death times of topological features in the filtration. This allows us to distinguish the features that are only noise and have very short lifespans from the more persistent ones. To compute these features and their lifespans, homology is applied to the filtration, which leads to a functor $\mathbb{R} \rightarrow$ Vect, which is often called a persistence module. There are two main visualizations of persistence modules: barcodes, which collect the birth and death times of homology classes in the filtration as intervals (see [18]); and persistence diagrams, which collect the same information as points in $\mathbb{R}^{2}$ (see [6] and [5]).

Since persistent homology is motivated by problems in data-analysis, we need to have a notion of distance between invariants obtained from different data sets, which must be stable with respect to noise in data. For barcodes and persistence diagrams, the bottleneck distance and the Wasserstein distances are the most commonly used distances. For persistence modules themselves, we have the interleaving distance,

Received February 2, 2018, revised September 11, 2018; published on November 20, 2019. 2010 Mathematics Subject Classification: 55U99.

Key words and phrases: persistence module, persistent homology.

Article available at http://dx.doi.org/10.4310/HHA.2020.v22.n1.a14

Copyright (C) 2019, Ville Puuska. Permission to copy for private use granted. 
which has been generalized to extensions of persistence modules, e.g. to multidimensional and generalized persistence modules (see [14] and [2]). For persistence modules $\mathbb{R} \rightarrow$ Vect, the interleaving distance is computable, because it is equal to the bottleneck distance. For multidimensional persistence modules, there are currently no efficient algorithms to compute the interleaving distance. In fact, it was recently shown by Bjerkevik and Botnan [1] that computing the distance for multidimensional persistence modules is at least as hard as a constrained matrix invertibility problem, and they conjectured that computing the distance is NP-hard.

In this paper, we present a stable distance for persistence modules $\mathbf{P} \rightarrow \mathbf{C}$, i.e. functors, which is computed directly from invariants of persistence modules known as rank invariants, where $\mathbf{P}$ is a preordered set and $\mathbf{C}$ is an Abelian category. This distance is an extension of two previous distances: the erosion distance of [16], and the distance $\mathrm{d}_{T}$ of $[\mathbf{1 1}]$. We call this distance the erosion distance after the former. We show that the erosion distance is stable with respect to the interleaving distance, and that it gives a lower bound for the natural pseudo-distance in the case of sublevel set persistent homology of continuous functions.

The distance $\mathrm{d}_{T}$ was introduced by Frosini in [11] as a distance for multidimensional persistent homology groups with torsion, i.e. persistence modules obtained by applying singular homology with coefficients in an Abelian group to a multiparameter filtration of a space. It was shown that $\mathrm{d}_{T}$ gives a lower bound for the natural pseudo-distance when the filtrations are obtained as sublevel set filtrations of continuous functions. This distance can be directly extended for all functors $\mathbb{R}^{n} \rightarrow \mathbf{A b}$.

A recent step forward in the effort to extend the theory of persistent homology came when Patel $[\mathbf{1 6}]$ generalized the persistence diagram for so called constructible persistence modules $\mathbb{R} \rightarrow \mathbf{C}$, where $\mathbf{C}$ is any essentially small symmetric monoidal category with images. Additionally, a new distance for persistence diagrams, the erosion distance, was introduced.

This paper has two main purposes. Firstly, we wish to extend the erosion distance of [16], independent of persistence diagrams, in order to allow it to be used in the multidimensional setting without requiring constructibility. Secondly, we wish to look at the distance $\mathrm{d}_{T}$ of $[\mathbf{1 1}]$ from a more categorical perspective. Essentially, defining either of these distances starts with giving a preorder of the target category $\mathbf{C}$, and then extending it to a preorder of maps $\operatorname{Dgm}_{\mathbb{R}^{n}}:=\left\{(a, b) \in \mathbb{R}^{n} \times \mathbb{R}^{n} \mid a<b\right\} \rightarrow \mathbf{C}$. Then, every persistence module $F: \mathbb{R}^{n} \rightarrow \mathbf{C}$ induces a map

$$
\mathcal{F}: \operatorname{Dgm}_{\mathbb{R}^{n}} \rightarrow \mathbf{C}, \mathcal{F}(a, b)=\operatorname{im} F(a<b),
$$

which is a straightforward generalization of the rank invariant of [4]. For maps $f, g: \operatorname{Dgm}_{\mathbb{R}^{n}} \rightarrow \mathbf{C}$, we get an extended pseudo-metric by taking the infimum of all $\varepsilon \in[0, \infty)$ such that

$$
f(a-\varepsilon, b+\varepsilon) \leqslant g(a, b) \text { and } g(a-\varepsilon, b+\varepsilon) \leqslant f(a, b) \text { for all }(a, b) \in \operatorname{Dgm}_{\mathbb{R}^{n}},
$$

which gives us an extended pseudo-metric for rank invariants of persistence modules $\mathbb{R}^{n} \rightarrow \mathbf{C}$. To extend this to persistence modules $\mathbf{P} \rightarrow \mathbf{C}$, we use translations of the preordered set $\mathbf{P}$ and superlinear families or sublinear projections in fundamentally the same way that they are used in [2], where they are used to extend the interleaving distance for generalized persistence modules. 


\section{Outline}

In section 2, we define the erosion distance in its most general form, i.e. for (decreasing) maps

$$
\operatorname{Dgm}_{\mathbf{P}}=\{(a, b) \in \mathbf{P} \times \mathbf{P} \mid a<b\} \rightarrow \mathbf{G},
$$

where $\mathbf{G}$ is a preordered class, and $\mathbf{P}$ is a preordered set equipped with a sublinear projection or a superlinear family. We also show in subsection 2.1 that the $L^{\infty}$ distance of functions $X \rightarrow \mathbb{R}$, where $X$ is any set can be interpreted as an erosion distance.

In section 3, we look at the erosion distance in full generality. We start by looking at suitable preorders for the target category $\mathbf{C}$ in subsection 3.1. Then, in subsection 3.2, we define the erosion distance for rank invariants of persistence modules. We prove that it is an extended pseudo-metric (Corollary 3.8), and that it is stable with respect to the interleaving distance (Theorem 3.11). In subsection 3.3, we go over the details of the erosion distance of Patel $[\mathbf{1 6}]$ and show that the two erosion distances are equal.

In section 4 , we show that the distance $\mathrm{d}_{T}$ of Frosini $[\mathbf{1 1}]$ is a special case of the erosion distance. First, in subsection 4.1 we define the subquotient preorder of $\mathbf{A b}$ that is implicitly defined in [11] and we define the distance $\mathrm{d}_{T}$. We show that $\mathrm{d}_{T}$ is equal to the erosion distance in Proposition 4.6. In subsection 4.2, we show that the erosion distance gives a lower bound for the natural pseudo-distance (Theorem 4.9).

In section 5, we consider the situation where $\mathbf{P}$ is equipped with a sublinear projection and a superlinear family. We show that if the sublinear projection and the superlinear family satisfy the adjunction relation as defined in $[\mathbf{2}]$, then the two erosion distances are equal.

\section{Acknowledgments}

The author thanks the anonymous reviewers for particularly helpful comments. The author acknowledges the support of the Jenny and Antti Wihuri Foundation grant number 00170307.

\section{Erosion distance for maps}

Throughout these notes we let $\mathbf{P}$ be a preordered set and $\mathbf{G}$ be a preordered class. We regard preordered sets and classes as categories by taking the elements of the category to be the elements of the set or class, and morphisms to be the relations $a \leqslant b$.

We denote ${ }^{1}$

$$
\operatorname{Dgm}_{\mathbf{P}}=\{(a, b) \in \mathbf{P} \times \mathbf{P} \mid a<b\}
$$

and we define a preorder for the set $\operatorname{Dgm}_{\mathbf{P}}$ by setting

$$
(a, b) \leqslant\left(a^{\prime}, b^{\prime}\right) \Longleftrightarrow a^{\prime} \leqslant a \text { and } b \leqslant b^{\prime},
$$

i.e. the preorder inherited from $\mathbf{P}^{\mathrm{op}} \times \mathbf{P}$. In other words, $(a, b) \leqslant\left(a^{\prime}, b^{\prime}\right)$, if and only if the interval $[a, b]$ is a subset of the interval $\left[a^{\prime}, b^{\prime}\right]$.

\footnotetext{
${ }^{1}$ We use the notation $a<b$ to mean that $a \leqslant b$ and $a \neq b$.
} 
Let $\mathbf{P}=\mathbb{R}$ and take a function $f: \operatorname{Dgm}_{\mathbb{R}} \rightarrow \mathbf{G}$. We can think of the function $f$ as an assignment of elements of $\mathbf{G}$ to each point in $\operatorname{Dgm}_{\mathbb{R}}$. Now, let $\varepsilon \geqslant 0$ and consider the function

$$
f_{\varepsilon}: \operatorname{Dgm}_{\mathbb{R}} \rightarrow \mathbf{G}, f_{\varepsilon}(a, b)=f(a-\varepsilon, b+\varepsilon) .
$$

We can think of the assignment of elements given by $f_{\varepsilon}$ as moving the points of $f$ down and right by $\varepsilon$, or towards the diagonal $\{(x, y) \mid x=y\}$ by $\sqrt{2} \varepsilon$, and killing elements that are moved to or below the diagonal. If $g: \operatorname{Dgm}_{\mathbb{R}} \rightarrow \mathbf{G}$ is another function, we can ask how much we need to move $f$ and $g$ towards the diagonal to get the pair of inequalities

$$
f_{\varepsilon} \leqslant g \text { and } g_{\varepsilon} \leqslant f .
$$

It's easy to see that by taking the infimum over all $\varepsilon$ such that these inequalities hold we get an extended pseudo-metric for functions $\operatorname{Dgm}_{\mathbb{R}} \rightarrow \mathbf{G}$. This idea can be generalized to arbitrary preordered sets $\mathbf{P}$ by using translations and superlinear families or sublinear projections in the same way as in [2]. Specifically, instead of moving points down and right by $\varepsilon$, we move them by a pair of translations of $\mathbf{P}$.

Definition 2.1. A translation of the set $\mathbf{P}$ is a map $\Gamma: \mathbf{P} \rightarrow \mathbf{P}$ such that:

i) $\Gamma$ is a bijection,

ii) $a \leqslant b \Rightarrow \Gamma a \leqslant \Gamma b$ and $\Gamma^{-1} a \leqslant \Gamma^{-1} b$ for all $a, b \in \mathbf{P}$,

iii) $a \leqslant \Gamma a$ for all $a \in \mathbf{P}$.

In other words, items i) and ii) say that $\Gamma$ is an automorphism of $\mathbf{P}$, and item iii) says that there exists a natural transformation $I \Rightarrow \Gamma$, where $I$ is the identity functor. We denote the preordered set of translations of $\mathbf{P}$ by $\operatorname{Trans}_{\mathbf{P}}$.

Note that our definition of a translation is stricter than the one in [2] since we require a translation to be an automorphism, instead of just an endofunctor of $\mathbf{P}$. Also note that Trans $\mathbf{P}$ is closed under composition and for every $\Gamma \in$ Trans $_{\mathbf{P}}$ we have $\Gamma^{-1} a \leqslant a$ for all $a \in \mathbf{P}$.

The following lemma will be used later on.

Lemma 2.2. For all $\Gamma, \mathrm{K} \in$ Trans $_{\mathrm{P}}$

$$
\Gamma \leqslant \mathrm{K} \Rightarrow \mathrm{K}^{-1} \leqslant \Gamma^{-1} .
$$

Proof. We assume that $\Gamma \leqslant \mathrm{K}$ and take $a \in \mathbf{P}$. Since $\Gamma$ has to be a bijection, we can take $b \in \mathbf{P}$ such that $a=\Gamma b$. Now

$$
\Gamma b \leqslant \mathrm{~K} b \quad \Rightarrow \quad K^{-1} \Gamma b \leqslant b \quad \Rightarrow \quad \mathrm{K}^{-1} \Gamma b \leqslant \Gamma^{-1} \Gamma b \quad \Rightarrow \quad \mathrm{K}^{-1} a \leqslant \Gamma^{-1} a .
$$

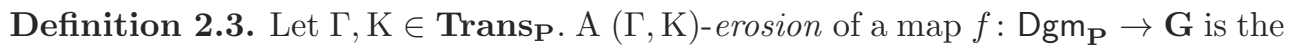
map

$$
\nabla_{\Gamma, \mathrm{K}} f: \operatorname{Dgm}_{\mathbf{P}} \rightarrow \mathbf{G},(a, b) \mapsto f\left(\Gamma^{-1} a, \mathrm{~K} b\right) .
$$

We also use the shorthand $\nabla_{\Gamma} f=\nabla_{\Gamma, \Gamma} f$.

Remark 2.4. In later sections, the function $f: \operatorname{Dgm}_{\mathbf{P}} \rightarrow \mathbf{G}$ will be the rank invariant of a persistence module, which is always decreasing. It would be natural to assume that $f$ is decreasing, since then we would have $\nabla_{\Gamma, \mathrm{K}} f \leqslant f$ for all $\Gamma, \mathrm{K} \in$ 
$\operatorname{Trans}_{\mathbf{P}}$, which matches the intuition that eroding a function decreases it. However, the assumption is needed only once in this section, so we do not assume it in general.

Proposition 2.5 (Triangle inequality). Let $f, g, h: \operatorname{Dgm}_{\mathbf{P}} \rightarrow \mathbf{G}$ and $\Gamma, \Gamma^{\prime}, \mathrm{K}, \mathrm{K}^{\prime} \in$ Transp such that

$$
\nabla_{\Gamma, \mathrm{K}} f \leqslant g, \nabla_{\mathrm{K}, \Gamma} g \leqslant f \text { and } \nabla_{\Gamma^{\prime}, \mathrm{K}^{\prime}} g \leqslant h, \nabla_{\mathrm{K}^{\prime}, \Gamma^{\prime}} h \leqslant g .
$$

Then

$$
\nabla_{\Gamma^{\prime} \Gamma, \mathrm{KK}^{\prime}} f \leqslant h, \nabla_{\mathrm{KK}^{\prime}, \Gamma^{\prime} \Gamma} h \leqslant f .
$$

Proof. Take $(a, b) \in \operatorname{Dgm}_{\mathbf{P}}$. Now

$$
f\left(\left(\Gamma^{\prime} \Gamma\right)^{-1} a, \mathrm{KK}^{\prime} b\right)=f\left(\Gamma^{-1} \Gamma^{\prime-1} a, \mathrm{KK}^{\prime} b\right) \leqslant g\left(\Gamma^{\prime-1} a, \mathrm{~K}^{\prime} b\right) \leqslant h(a, b),
$$

so $\nabla_{\Gamma^{\prime} \Gamma, \mathrm{KK}^{\prime}} f(a, b) \leqslant h(a, b)$, and

$$
h\left(\left(\mathrm{KK}^{\prime}\right)^{-1} a, \Gamma^{\prime} \Gamma b\right)=h\left(\mathrm{~K}^{\prime-1} \mathrm{~K}^{-1} a, \Gamma^{\prime} \Gamma b\right) \leqslant g\left(\mathrm{~K}^{-1} a, \Gamma b\right) \leqslant f(a, b),
$$

so $\nabla_{\mathrm{KK}^{\prime}, \Gamma^{\prime} \Gamma} h(a, b) \leqslant f(a, b)$.

Definition $2.6([2])$. A function $\Omega:[0, \infty) \rightarrow \operatorname{Trans}_{\mathbf{P}}$ is called a superlinear family if for all $\varepsilon, \varepsilon^{\prime} \in[0, \infty)$

$$
\Omega_{\varepsilon} \Omega_{\varepsilon^{\prime}} \leqslant \Omega_{\varepsilon+\varepsilon^{\prime}}
$$

An increasing function $\omega$ : Trans $\mathbf{P} \rightarrow[0, \infty]$ is called a sublinear projection if $\omega_{I}=0$, where $I$ is the identity translation on $\mathbf{P}$, and for all $\Gamma, K \in \operatorname{Trans}_{\mathbf{P}}$

$$
\omega_{\Gamma \mathrm{K}} \leqslant \omega_{\Gamma}+\omega_{\mathrm{K}}
$$

Note that a superlinear family is always increasing, since for $\varepsilon \leqslant \varepsilon^{\prime}$

$$
\Omega_{\varepsilon}=I \Omega_{\varepsilon} \leqslant \Omega_{\varepsilon^{\prime}-\varepsilon} \Omega_{\varepsilon} \leqslant \Omega_{\varepsilon^{\prime}}
$$

Hence, a superlinear family is a functor $[0, \infty) \rightarrow$ Trans $_{\mathbf{P}}$ and a sublinear projection is functor $\operatorname{Trans}_{\mathbf{P}} \rightarrow[0, \infty]$. Note also that we require $\omega_{I}=0$, but we do not require $\Omega_{0}=I$.

Definition 2.7 (Erosion distance).

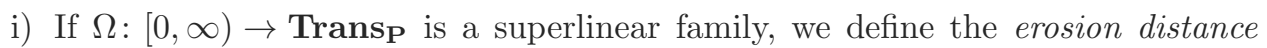
w.r.t. $\Omega$ for maps $f, g: \operatorname{Dgm}_{\mathbf{P}} \rightarrow \mathbf{G}$ to be ${ }^{2}$

$$
\mathrm{d}_{E}^{\Omega}(f, g)=\inf \left\{\varepsilon \mid \nabla_{\Omega_{\varepsilon}} f \leqslant g, \nabla_{\Omega_{\varepsilon}} g \leqslant f\right\} .
$$

ii) If $\omega: \operatorname{Trans}_{\mathbf{P}} \rightarrow[0, \infty]$ is a sublinear projection, we define the erosion distance w.r.t. $\omega$ for maps $f, g: \operatorname{Dgm}_{\mathbf{P}} \rightarrow \mathbf{G}$ to be

$$
\mathrm{d}_{E}^{\omega}(f, g)=\inf \left\{\varepsilon \mid \exists \Gamma, \mathrm{K} \text { such that } \omega_{\Gamma}, \omega_{\mathrm{K}} \leqslant \varepsilon \text { and } \nabla_{\Gamma, \mathrm{K}} f \leqslant g, \nabla_{\mathrm{K}, \Gamma} g \leqslant f\right\} .
$$

If the choice of $\Omega$ or $\omega$ is clear from context, we use a shorthand notation $d_{E}$ for the erosion distance.

\footnotetext{
${ }^{2}$ In this paper, we use the convention that inf $\emptyset=\infty$.
} 
Example 2.8. Let $\mathbf{P}=\mathbb{R}, \mathbf{G}=\mathbb{N}$, and $\Omega_{\varepsilon}(a)=a+\varepsilon$. Let $f, g: \operatorname{Dgm}_{\mathbb{R}} \rightarrow \mathbb{N}$

$$
f(a, b)= \begin{cases}2, & \text { if }[a, b] \subseteq[0,1], \\ 1, & \text { if }[a, b] \subseteq[0,2] \text { and }[a, b] \nsubseteq[0,1], \\ 0, & \text { otherwise, }\end{cases}
$$

and

$$
g(a, b)= \begin{cases}2, & \text { if }[a, b] \subseteq[1,2] \\ 1, & \text { if }[a, b] \subseteq[0,2] \text { and }[a, b] \nsubseteq[1,2] \\ 0, & \text { otherwise }\end{cases}
$$
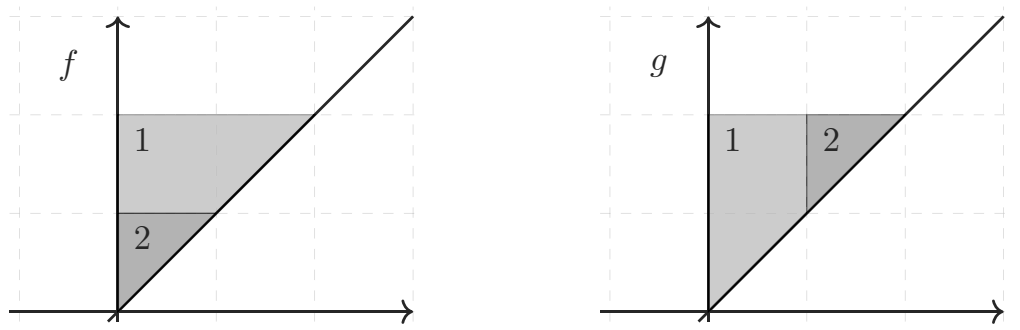

Figure 1: The functions $f$ and $g$ of Example 2.8.

Let $\varepsilon \in[0,1 / 2)$, and $\delta=1 / 2-\varepsilon>0$. Now, we have $(1 / 2-\delta, 1 / 2) \in \operatorname{Dgm}_{\mathbb{R}}$ and

$$
\nabla_{\Omega_{\varepsilon}} f(1 / 2-\delta, 1 / 2)=f(1 / 2-\delta-\varepsilon, 1 / 2+\varepsilon)=2 \not 1=g(1 / 2-\delta, 1 / 2) .
$$

Hence $\nabla_{\Omega_{\varepsilon}} f \nless k g$. Similarly $\nabla_{\Omega_{\varepsilon}} g \nless f$. For $\varepsilon \geqslant 1 / 2$, we have $\nabla_{\Omega_{\varepsilon}} f \leqslant g$ and $\nabla_{\Omega_{\varepsilon}} g \leqslant f$. Hence $\mathrm{d}_{E}^{\Omega}(f, g)=1 / 2$.

\section{Proposition 2.9.}

i) If $\Omega:[0, \infty) \rightarrow$ Trans $\mathbf{P}$ is a superlinear family, then $\mathrm{d}_{E}^{\Omega}$ is an extended pseudometric on the set of decreasing functions $\operatorname{Dgm}_{\mathbf{P}} \rightarrow \mathbf{G}$.

ii) If $\Omega:[0, \infty) \rightarrow$ Trans $_{\mathbf{P}}$ is linear, i.e. $\Omega_{0}=I$ and $\Omega_{a} \Omega_{b}=\Omega_{a+b}$ for all $a, b \in$ $[0, \infty)$, then $\mathrm{d}_{E}^{\Omega}$ is an extended pseudo-metric on the set of all functions $\operatorname{Dgm}_{\mathbf{P}} \rightarrow$ G.

iii) If $\omega:$ Trans $\mathbf{P} \rightarrow[0, \infty]$ is a sublinear projection, then $\mathrm{d}_{E}^{\omega}$ is an extended pseudometric on the set of all functions $\operatorname{Dgm}_{\mathbf{P}} \rightarrow \mathbf{G}$.

Proof. It's trivial that $\mathrm{d}_{E}^{\Omega}$ and $\mathrm{d}_{E}^{\omega}$ are symmetric and non-negative in all cases. Additionally, in cases ii) and iii) it's clear that $\mathrm{d}_{E}(f, f)=0$ for all maps $f: \operatorname{Dgm}_{\mathbf{P}} \rightarrow \mathbf{G}$. If we take a decreasing map $f: \operatorname{Dgm}_{\mathbf{P}} \rightarrow \mathbf{G}$, we see that $\nabla_{\Gamma, \mathrm{K}} f \leqslant f$ for all $\Gamma, \mathrm{K} \in$ Trans, so, in particular, $\nabla_{\Omega_{0}} f \leqslant f$. This implies that $\mathrm{d}_{E}^{\Omega}(f, f)=0$. All that remains to prove is the triangle inequality.

i) Let $f, g, h: \operatorname{Dgm}_{\mathbf{P}} \rightarrow \mathbf{G}$ and $\varepsilon, \varepsilon^{\prime} \geqslant 0$ such that

$$
\nabla_{\Omega_{\varepsilon}} f \leqslant g, \nabla_{\Omega_{\varepsilon}} g \leqslant f, \nabla_{\Omega_{\varepsilon^{\prime}}} g \leqslant h, \nabla_{\Omega_{\varepsilon^{\prime}}} h \leqslant g .
$$

By the triangle inequality (proposition 2.5) $\nabla_{\Omega_{\varepsilon^{\prime}} \Omega_{\varepsilon}} f \leqslant h$ and $\nabla_{\Omega_{\varepsilon} \Omega_{\varepsilon^{\prime}}} h \leqslant f$. 
We assume that $f$ and $h$ are decreasing and take $(a, b) \in \operatorname{Dgm}_{\mathbf{P}}$. We notice that by superlinearity and Lemma $2.2\left(\left(\Omega_{\varepsilon^{\prime}} \Omega_{\varepsilon}\right)^{-1} a, \Omega_{\varepsilon^{\prime}} \Omega_{\varepsilon} b\right) \leqslant\left(\Omega_{\varepsilon+\varepsilon^{\prime}}^{-1} a, \Omega_{\varepsilon+\varepsilon^{\prime}} b\right)$. Since $f$ is decreasing

$$
\nabla_{\Omega_{\varepsilon+\varepsilon^{\prime}}} f(a, b)=f\left(\Omega_{\varepsilon+\varepsilon^{\prime}}^{-1} a, \Omega_{\varepsilon+\varepsilon^{\prime}} b\right) \leqslant f\left(\left(\Omega_{\varepsilon^{\prime}} \Omega_{\varepsilon}\right)^{-1} a, \Omega_{\varepsilon^{\prime}} \Omega_{\varepsilon} b\right)=\nabla_{\Omega_{\varepsilon^{\prime}} \Omega_{\varepsilon}} f(a, b) .
$$

Hence $\nabla_{\Omega_{\varepsilon+\varepsilon^{\prime}}} f \leqslant h$. Similarly, we see that $\nabla_{\Omega_{\varepsilon+\varepsilon^{\prime}}} h \leqslant f$.

ii) Let $f, g, h$ and $\varepsilon, \varepsilon^{\prime}$ be as in the previous case. By the same argument, $\nabla_{\Omega_{\varepsilon^{\prime}} \Omega_{\varepsilon}} f \leqslant$ $h$ and $\nabla_{\Omega_{\varepsilon} \Omega_{\varepsilon^{\prime}}} h \leqslant f$. If $\Omega$ is linear, these inequalities give us $\nabla_{\Omega_{\varepsilon+\varepsilon^{\prime}}} f \leqslant h$ and $\nabla_{\Omega_{\varepsilon+\varepsilon^{\prime}}} h \leqslant f$.

iii) Let $f, g, h: \operatorname{Dgm}_{\mathbf{P}} \rightarrow \mathbf{G}, \varepsilon, \varepsilon^{\prime} \geqslant 0$ and $\Gamma, \Gamma^{\prime}, \mathrm{K}, \mathrm{K}^{\prime} \in$ Trans such that $\omega_{\Gamma}, \omega_{\mathrm{K}} \leqslant$ $\varepsilon, \omega_{\Gamma^{\prime}}, \omega_{\mathrm{K}^{\prime}} \leqslant \varepsilon^{\prime}$ and

$$
\nabla_{\Gamma, \mathrm{K}} f \leqslant g, \nabla_{\mathrm{K}, \Gamma} g \leqslant f, \nabla_{\Gamma^{\prime}, \mathrm{K}^{\prime}} g \leqslant h, \nabla_{\mathrm{K}^{\prime}, \Gamma^{\prime}} h \leqslant g .
$$

By the triangle inequality (proposition 2.5)

$$
\nabla_{\Gamma^{\prime} \Gamma, \mathrm{KK}^{\prime}} f \leqslant h, \nabla_{\mathrm{KK}^{\prime}, \Gamma^{\prime} \Gamma} h \leqslant f,
$$

and by sublinearity $\omega_{\Gamma^{\prime} \Gamma}, \omega_{\mathrm{KK}} \leqslant \varepsilon+\varepsilon^{\prime}$.

\subsection{The $L^{\infty}$-distance as an erosion distance}

As our first example, we consider the erosion distance of interlevel set filtrations of functions $f: X \rightarrow \mathbb{R}$, where $X$ is a fixed set. We show that this is simply the $L^{\infty}$-distance $\mathrm{d}_{\infty}(f, g)=\|f-g\|_{\infty}$ of functions $f, g: X \rightarrow \mathbb{R}$.

Definition 2.10. Let $X$ be a set. To every function $f: X \rightarrow \mathbb{R}$ we attach the interlevel set function

$$
F: \operatorname{Dgm}_{\mathbb{R}} \rightarrow \text { Set, } F(a, b)=f^{-1}([a, b]),
$$

where Set is the category of sets.

Let $\Omega:[0, \infty) \rightarrow \operatorname{Trans}_{\mathbb{R}}, \Omega_{\varepsilon}(a)=a+\varepsilon$. We define a preorder for Set by taking the opposite of the natural preorder of sets, i.e. we set

$$
A \leqslant B \Longleftrightarrow A \supseteq B
$$

Theorem 2.11. Let $f, g: X \rightarrow \mathbb{R}$ be functions, and let $F, G: \operatorname{Dgm}_{\mathbb{R}} \rightarrow$ Set be the interlevel set functions. Then

$$
\mathrm{d}_{\infty}(f, g)=\mathrm{d}_{E}^{\Omega}(F, G) .
$$

Proof. Let $f, g: X \rightarrow \mathbb{R}$ and $\varepsilon \in[0, \infty)$. Now,

$$
\begin{aligned}
d_{\infty}(f, g) \leqslant \varepsilon & \Longleftrightarrow g(x)-\varepsilon \leqslant f(x) \leqslant g(x)+\varepsilon \text { for all } x \in X \\
& \Longleftrightarrow g^{-1}([r, r]) \subseteq f^{-1}([r-\varepsilon, r+\varepsilon]) \text { for all } r \in \mathbb{R} \\
& \Longleftrightarrow g^{-1}([a, b]) \subseteq f^{-1}([a-\varepsilon, b+\varepsilon]) \text { for all } a \leqslant b \in \mathbb{R} \\
& \Longleftrightarrow g^{-1}([a, b]) \subseteq f^{-1}([a-\varepsilon, b+\varepsilon]) \text { for all } a<b \in \mathbb{R} \\
& \Longleftrightarrow \nabla_{\Omega_{\varepsilon}} F \leqslant G .
\end{aligned}
$$

To see the second equivalence, set $r=g(x)$, and to see the $\Leftarrow$ direction of the second 
to last equivalence, note that

$$
\begin{aligned}
g^{-1}([r, r]) & =g^{-1}\left(\bigcap_{i=1}^{\infty}\left[r-\frac{1}{n}, r\right]\right) \\
& =\bigcap_{i=1}^{\infty} g^{-1}\left(\left[r-\frac{1}{n}, r\right]\right) \\
& \subseteq \bigcap_{i=1}^{\infty} f^{-1}\left(\left[r-\frac{1}{n}-\varepsilon, r+\varepsilon\right]\right) \\
& =f^{-1}\left(\bigcap_{i=1}^{\infty}\left[r-\frac{1}{n}-\varepsilon, r+\varepsilon\right]\right) \\
& =f^{-1}([r-\varepsilon, r+\varepsilon])
\end{aligned}
$$

for all $r \in \mathbb{R}$. By symmetry of the first inequality, we get $\mathrm{d}_{\infty}(f, g) \leqslant \varepsilon \Longleftrightarrow \Omega_{\varepsilon} G \leqslant F$. Hence,

$$
\inf \left\{\varepsilon \mid \mathrm{d}_{\infty}(f, g) \leqslant \varepsilon\right\}=\inf \left\{\varepsilon \mid \nabla_{\Omega_{\varepsilon}} G \leqslant F \text { and } \nabla_{\Omega_{\varepsilon}} F \leqslant G\right\},
$$

i.e. $\mathrm{d}_{\infty}(f, g)=\mathrm{d}_{E}^{\Omega}(F, G)$.

\section{Erosion distance for persistence modules}

In this section, we specialize the erosion distance for rank invariants of persistence modules $\mathbf{P} \rightarrow \mathbf{C}$, where $\mathbf{P}$ is a preordered set and $\mathbf{C}$ is an Abelian category with a suitable preorder for its objects. First, in subsection 3.1 we consider which preorders for an Abelian category $\mathbf{C}$ are suitable. In subsection 3.2 we define the distance in full generality, and finally, in subsection 3.3 we go over the details of the erosion distance of Patel $[\mathbf{1 6}]$ and show that the distances are equal.

\subsection{Preorders that respect mono- and epimorphisms}

If we have a sublinear projection $\omega$, or a linear family $\Omega$, Proposition 2.9 shows that this information is enough to make the erosion distance an extended pseudo-metric for functions $\operatorname{Dgm}_{\mathbf{P}} \rightarrow \mathbf{C}$. However, if we have a superlinear family that is not linear, we need to restrict ourselves to decreasing functions. To make sure that the functions induced by persistence modules, i.e. the rank invariants, are indeed decreasing, we first need to consider which preorders of $\mathbf{C}$ are suitable. A natural idea is to require objects to be larger than their subobjects and quotients, and this turns out to be enough.

Lemma 3.1. Let $\mathbf{C}$ be an Abelian category equipped with a preorder $\leqslant$ for its objects such that for all $A, B \in \mathbf{C}$

$$
A \hookrightarrow B \Rightarrow A \leqslant B
$$

and

$$
A \rightarrow B \Rightarrow A \geqslant B
$$

Then, for all morphisms $f: A \rightarrow B$ :

i) $\operatorname{ker} f \leqslant A$ and coker $f \leqslant B$, 
ii) $\operatorname{im} f \leqslant A, B$,

iii) if $f$ is an isomorphism, then $A \leqslant B$ and $B \leqslant A$.

Additionally, every preorder that satisfies condition i) also satisfies

$$
A \hookrightarrow B \Rightarrow A \leqslant B
$$

and

$$
A \rightarrow B \Rightarrow A \geqslant B \text {. }
$$

Proof. Cases i)-iii) are trivial. The last remark follows from the fact that in an Abelian category every monomorphism is a kernel morphism and every epimorphism is a cokernel morphism.

Definition 3.2. A preorder of an Abelian category $\mathbf{C}$ that satisfies the conditions in the previous lemma is said to respect mono- and epimorphisms.

Example 3.3. For the category of finite dimensional vector spaces, we get a preorder that respects mono- and epimorphisms by setting

$$
A \leqslant B \Longleftrightarrow \operatorname{dim} A \leqslant \operatorname{dim} B .
$$

Lemma 3.4. Let $\mathbf{C}$ be an Abelian category equipped with a preorder that respects mono- and epimorphisms. Let $f: A \rightarrow B, g: A^{\prime} \rightarrow A, h: B \rightarrow B^{\prime}$ be morphisms in $\mathbf{C}$ and denote $f^{\prime}=h f g$. Then

$$
\operatorname{im} f^{\prime} \leqslant \operatorname{im} f .
$$

Proof. Note that $f^{\prime}$ factors as $A^{\prime} \stackrel{\operatorname{coker}\left(\operatorname{ker} f^{\prime}\right)}{\longrightarrow} \operatorname{im} f^{\prime} \hookrightarrow B^{\prime}$ (see [15, VIII.3]). If we can factor $f^{\prime}$ as $A^{\prime} \stackrel{\varphi}{\rightarrow} I \stackrel{\psi}{\longrightarrow} B^{\prime}$, then the diagram

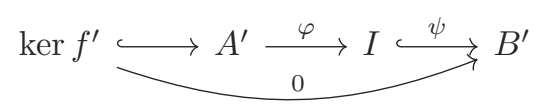

commutes. Since $\psi$ is a monomorphism, we get that the diagram

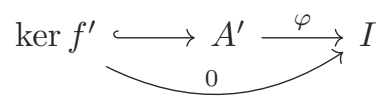

commutes. Hence, we can fill in the commutative diagram

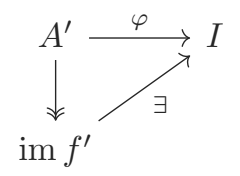

Since $A^{\prime} \rightarrow \operatorname{im} f^{\prime}$ is an epimorphism, the diagram

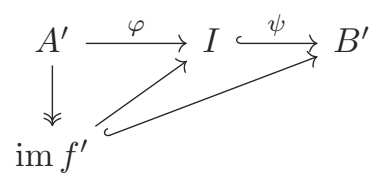

commutes. Finally, since $\operatorname{im} f^{\prime} \longrightarrow I \leftrightharpoons B^{\prime}$ commutes, the map im $f^{\prime} \rightarrow I$ has to be a monomorphism. 
By using the above result to the commutative diagram

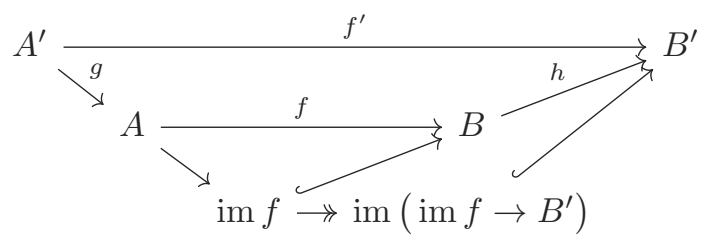

we get $\operatorname{im} f^{\prime} \hookrightarrow \operatorname{im}\left(\operatorname{im} f \rightarrow B^{\prime}\right)$. Hence

$$
\operatorname{im} f^{\prime} \leqslant \operatorname{im}\left(\operatorname{im} f \rightarrow B^{\prime}\right) \leqslant \operatorname{im} f .
$$

\subsection{Erosion distance for persistence modules}

In this subsection we define the rank invariant and the erosion distance for persistence modules $\mathbf{P} \rightarrow \mathbf{C}$, where $\mathbf{P}$ is a preordered set and $\mathbf{C}$ is an Abelian category with a preorder that respects mono- and epimorphisms. We also show that the erosion distance is stable with respect to the interleaving distance of $[\mathbf{2}]$.

Definition 3.5 (Rank invariant and erosion distance). To every persistence module $F: \mathbf{P} \rightarrow \mathbf{C}$, i.e. a functor, we attach a map $\mathcal{F}: \operatorname{Dgm}_{\mathbf{P}} \rightarrow \mathbf{C}$ by setting for each $(a, b) \in$ $\operatorname{Dgm}_{\mathbf{P}}$

$$
\mathcal{F}(a, b)=\operatorname{im} F(a<b) .
$$

We call this map the rank invariant of $F$. In addition, let $\Omega:[0, \infty) \rightarrow$ Trans $_{\mathbf{P}}$ be a superlinear family or $\omega$ : Trans $\mathbf{P} \rightarrow[0, \infty]$ be a sublinear projection. We define the erosion distance $\mathrm{d}_{E}$ of a pair of persistence modules $F, G: \mathbf{P} \rightarrow \mathbf{C}$ to be

$$
\mathrm{d}_{E}^{\Omega}(F, G)=\mathrm{d}_{E}^{\Omega}(\mathcal{F}, \mathcal{G})
$$

or

$$
\mathrm{d}_{E}^{\omega}(F, G)=\mathrm{d}_{E}^{\omega}(\mathcal{F}, \mathcal{G}) .
$$

Example 3.6. Take $\mathbf{P}=\mathbb{R}$, and let $\mathbf{k}$ be a field and $\mathbf{C}$ be the category of finite dimensional k-vector spaces equipped with the preorder $A \leqslant B \Longleftrightarrow \operatorname{dim} A \leqslant \operatorname{dim} B$. For every interval $I \subseteq \mathbb{R}$, we have a persistence module $C_{I}$, where

$$
C_{I}(a)= \begin{cases}\mathbf{k}, & \text { if } a \in I, \\ 0, & \text { otherwise }\end{cases}
$$

for all $a \in \mathbb{R}$, and the linear maps $C_{I}(a) \rightarrow C_{I}(b)$ are the identity where possible. Let $F=C_{[0,1]} \oplus C_{[0,2]}$ and $G=C_{[1,2]} \oplus C_{[0,2]}$. The rank invariants of $F$ and $G$ are essentially the functions $f$ and $g$ of Example 2.8. Hence, if we take $\Omega:[0, \infty) \rightarrow$ $\operatorname{Trans}_{\mathbb{R}}$ to be the standard superlinear family $\Omega_{\varepsilon}(a)=a+\varepsilon$, we have already seen in Example 2.8 that $\mathrm{d}_{E}^{\Omega}(F, G)=1 / 2$.

Proposition 3.7. For every persistence module $F: \mathbf{P} \rightarrow \mathbf{C}$ the map $\mathcal{F}$ is decreasing.

Proof. Let $(a, b) \leqslant\left(a^{\prime}, b^{\prime}\right)$, i.e. $a^{\prime} \leqslant a<b \leqslant b^{\prime}$. Since

$$
F\left(a^{\prime}<b^{\prime}\right)=F\left(b \leqslant b^{\prime}\right) F(a<b) F\left(a^{\prime} \leqslant a\right),
$$

Lemma 3.4 says that

$$
\mathcal{F}\left(a^{\prime}, b^{\prime}\right)=\operatorname{im} F\left(a^{\prime}<b^{\prime}\right) \leqslant \operatorname{im} F(a<b)=\mathcal{F}(a, b) .
$$


Corollary 3.8. The erosion distances $\mathrm{d}_{E}^{\Omega}$ and $\mathrm{d}_{E}^{\omega}$ are extended pseudo-metrics for persistence modules.

Proof. The claim follows directly from Proposition 3.7 and Proposition 2.9 i) and iii).

Now that we have shown that the erosion distances are extended pseudo-metrics, we'll consider stability with respect to the interleaving distance introduced in [2].

Definition 3.9 ([2]). Let $\Gamma, \mathrm{K} \in$ Trans $_{\mathbf{P}}$ and let $F, G: \mathbf{P} \rightarrow \mathbf{C}$ be persistence modules. A $(\Gamma, \mathrm{K})$-interleaving between $F$ and $G$ is a pair of natural transformations $(\varphi, \psi)$

$$
\varphi: F \Rightarrow G \Gamma, \psi: G \Rightarrow F \mathrm{~K}
$$

such that the following diagrams commute:
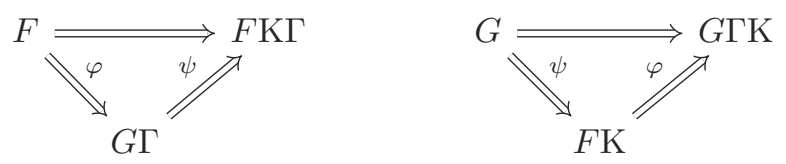

We say that $F$ and $G$ are $\varepsilon$-interleaved with respect to $\Omega$ if they are $\left(\Omega_{\varepsilon}, \Omega_{\varepsilon}\right)$-interleaved, and similarly that they are $\varepsilon$-interleaved with respect to $\omega$ if there exist $\Gamma, \mathrm{K} \in$ Trans ${ }_{\mathbf{P}}$ such that $\omega_{\Gamma}, \omega_{\mathrm{K}} \leqslant \varepsilon$ and $F$ and $G$ are $(\Gamma, \mathrm{K})$-interleaved.

We define the interleaving distances $\mathrm{d}_{I}^{\Omega}$ and $\mathrm{d}_{I}^{\omega}$ by setting

$$
\begin{aligned}
& \mathrm{d}_{I}^{\Omega}(F, G)=\inf \{\varepsilon \mid F \text { and } G \text { are } \varepsilon \text {-interleaved w.r.t. } \Omega\}, \\
& \mathrm{d}_{I}^{\omega}(F, G)=\inf \{\varepsilon \mid F \text { and } G \text { are } \varepsilon \text {-interleaved w.r.t. } \omega\} .
\end{aligned}
$$

Remark 3.10. Note that since in [2] a translation of $\mathbf{P}$ is not required to be an automorphism, and instead is only required to be an endofunctor of $\mathbf{P}$ with a natural transformation from the identity functor, our definition of the interleaving distance is slightly different. Specifically, our definition of $\mathrm{d}_{I}^{\Omega}$ is precisely the same, but for us the choice of $\Omega$ is more restricted, and our definition of $\mathrm{d}_{I}^{\omega}$ may be larger than the distance defined in [2].

Theorem 3.11 (Stability of the erosion distance). Let $F, G: \mathbf{P} \rightarrow \mathbf{C}$ be persistence modules. Then

$$
\mathrm{d}_{E}(F, G) \leqslant \mathrm{d}_{I}(F, G),
$$

where either $\mathrm{d}_{E}=\mathrm{d}_{E}^{\Omega}$ and $\mathrm{d}_{I}=\mathrm{d}_{I}^{\Omega}$, or $\mathrm{d}_{E}=\mathrm{d}_{E}^{\omega}$ and $\mathrm{d}_{I}=\mathrm{d}_{I}^{\omega}$.

Proof. To prove the claim in both cases, it is enough to show that if we have a $(\Gamma, \mathrm{K})$-interleaving between $F$ and $G$, then

$$
\nabla_{\Gamma, \mathrm{K}} \mathcal{F} \leqslant \mathcal{G}, \nabla_{\mathrm{K}, \Gamma} \mathcal{G} \leqslant \mathcal{F} .
$$

Let $(\varphi, \psi)$ be a $(\Gamma, \mathrm{K})$-interleaving between $F$ and $G$. For every $(a, b) \in \operatorname{Dgm}_{\mathbf{P}}$ we get 
a commutative diagram

$$
F\left(\Gamma^{-1} a\right) \underset{\underbrace{}_{G}}{\longrightarrow} F(\mathrm{~K} a) \underset{\psi>}{\longrightarrow} F(\mathrm{~K} b)
$$

This shows that

$$
F\left(\Gamma^{-1} a<\mathrm{K} b\right)=\psi_{b} \circ G(a<b) \circ \varphi_{\Gamma^{-1} a},
$$

and then by Lemma 3.4

$$
\nabla_{\Gamma, \mathrm{K}} \mathcal{F}(a, b)=\operatorname{im} F\left(\Gamma^{-1} a<\mathrm{K} b\right) \leqslant \operatorname{im} G(a<b)=\mathcal{G}(a, b) .
$$

Hence $\nabla_{\Gamma, \mathrm{K}} \mathcal{F} \leqslant \mathcal{G}$. Similarly, we can show that $\nabla_{\mathrm{K}, \Gamma} \mathcal{G} \leqslant \mathcal{F}$.

\subsection{Preorder induced by the Grothendieck group}

The main contribution of $[\mathbf{1 6}]$ is a generalization of persistence diagrams to constructible persistence modules over $\mathbb{R}$ with values in a category $\mathbf{C}$, where $\mathbf{C}$ is an essentially small symmetric monoidal category with images. The paper also introduces a distance for persistence diagrams, the erosion distance. In this subsection, we go over the details of the erosion distance of $[\mathbf{1 6}]$ and show that it is equal to our more general erosion distance.

Definition 3.12. Let $\mathbf{C}$ be an essentially small symmetric monoidal category with images.

i) We denote the set of isomorphism classes of $\mathbf{C}$ by $\mathcal{J}(\mathbf{C})$, and we make $\mathcal{J}(\mathbf{C})$ into a commutative monoid by setting

$$
[A]+[B]=[A \otimes B]
$$

for all $A, B \in \mathbf{C}$.

ii) $\mathcal{A}(\mathbf{C})$ is the group obtained by taking the group completion of $\mathcal{J}(\mathbf{C})$.

iii) If $\mathbf{C}$ happens to be Abelian, we consider $\mathbf{C}$ to be monoidal by taking the tensor product to be the coproduct $\otimes=\oplus \cdot \mathcal{B}(\mathbf{C})$ is the group obtained from $\mathcal{A}(\mathbf{C})$ by adding relations $[A]+[C]=[B]$ for all exact sequences $0 \rightarrow A \rightarrow B \rightarrow C \rightarrow 0$.

Both groups, $\mathcal{A}(\mathbf{C})$ and $\mathcal{B}(\mathbf{C})$, are often called the Grothendieck group of $\mathbf{C}$.

Example 3.13. Take $\mathbf{C}$ to be the category of finite dimensional vector spaces over a field $\mathbf{k}$. It is easy to see that $\mathcal{J}(\mathbf{C}) \cong \mathbb{N}$ and $\mathcal{A}(\mathbf{C}) \cong \mathcal{B}(\mathbf{C}) \cong \mathbb{Z}$.

Definition 3.14. A persistence module $F: \mathbb{R} \rightarrow \mathbf{C}$ is said to be constructible, if there exists a finite set $S=\left\{s_{1}, \ldots, s_{n}\right\} \subseteq \mathbb{R}$, where $s_{1}<s_{2}<\cdots<s_{n}$, such that:

- for $p \leqslant q<s_{1}$ the morphism $F(p \leqslant q)=\operatorname{id}_{e}$, where $e \in \mathbf{C}$ is the neutral element of the monoidal category,

- for $s_{i} \leqslant p \leqslant q<s_{i+1}$ the morphism $F(p \leqslant q)$ is an isomorphism, and

- for $s_{n} \leqslant p \leqslant q$ the morphism $F(p \leqslant q)$ is an isomorphism. 
Now, to every constructible persistence module $F: \mathbb{R} \rightarrow \mathbf{C}$ we attach a map

$$
d F_{\mathcal{A}}: \operatorname{Dgm}_{\mathbb{R}} \rightarrow \mathcal{A}(\mathbf{C}), d F_{\mathcal{A}}(a, b)=[\operatorname{im} F(a<b-\delta)],
$$

where $\delta>0$ is small enough so that $\operatorname{im} F\left(a<b-\delta^{\prime}\right) \cong \operatorname{im} F(a<b-\delta)$ for all $0<$ $\delta^{\prime}<\delta$. If $\mathbf{C}$ is Abelian, we attach a second map to $F$

$$
d F_{\mathcal{B}}: \operatorname{Dgm}_{\mathbb{R}} \rightarrow \mathcal{B}(\mathbf{C}), d F_{\mathcal{B}}(a, b)=[\operatorname{im} F(a<b-\delta)],
$$

where $\delta>0$ is again sufficiently small.

Theorem 3.15 ([16, Theorem 4.1]). The maps $d F_{\mathcal{A}}$ and $d F_{\mathcal{B}}$ have Möbius inversions, i.e. functions $F_{\mathcal{A}}: \operatorname{Dgm}_{\mathbb{R}} \rightarrow \mathcal{A}(\mathbf{C})$ and $F_{\mathcal{B}}: \operatorname{Dgm}_{\mathbb{R}} \rightarrow \mathcal{B}(\mathbf{C})$ with finite support such that

$$
\sum_{\mathbf{x} \geqslant \mathbf{a}} F_{\mathcal{A}}(\mathbf{x})=d F_{\mathcal{A}}(\mathbf{a}) \text { and } \sum_{\mathbf{x} \geqslant \mathbf{a}} F_{\mathcal{B}}(\mathbf{x})=d F_{\mathcal{B}}(\mathbf{a})
$$

for all $\mathbf{a} \in \operatorname{Dgm}_{\mathbb{R}}$.

The functions $F_{\mathcal{A}}$ and $F_{\mathcal{B}}$ are called the type $\mathcal{A}$ and type $\mathcal{B}$ persistence diagrams of $F$. Since in this article we always assume that $\mathbf{C}$ is Abelian, we will only focus on the type $\mathcal{B}$ diagrams.

Definition 3.16. We define a preorder for the Grothedieck group $\mathcal{B}(\mathbf{C})$ by setting $x \leqslant y \Longleftrightarrow$ there exists $A \in \mathbf{C}$ such that $x+[A]=y$.

This gives a preorder for $\mathbf{C}$

$$
A \leqslant B \Longleftrightarrow[A] \leqslant[B]
$$

The type $\mathcal{B}$ persistence diagrams of constructible persistence modules are preordered by setting for all constructible $F, G: \mathbb{R} \rightarrow \mathbf{C}$

$$
F_{\mathcal{B}} \preceq G_{\mathcal{B}} \Longleftrightarrow \sum_{\mathbf{x} \geqslant \mathbf{a}} F_{\mathcal{B}}(\mathbf{x}) \leqslant \sum_{\mathbf{x} \geqslant \mathbf{a}} G_{\mathcal{B}}(\mathbf{x}) \text { for all } \mathbf{a} \in \mathrm{Dgm}_{\mathbb{R}} .
$$

Since $F_{\mathcal{B}}$ and $G_{\mathcal{B}}$ are Möbius inversions of $d F_{\mathcal{B}}$ and $d G_{\mathcal{B}}$, this is equivalent to

$$
d F_{\mathcal{B}}(\mathbf{a}) \leqslant d G_{\mathcal{B}}(\mathbf{a}) \text { for all } \mathbf{a} \in \operatorname{Dgm}_{\mathbb{R}} .
$$

Example 3.1\%. Take $\mathbf{C}$ to be the category of finite dimensional vector spaces over a field $\mathbf{k}$ like in Example 3.13. The preorder of $\mathbf{C}$ we get from the previous definition is simply $A \leqslant B \Longleftrightarrow \operatorname{dim} A \leqslant \operatorname{dim} B$.

Definition 3.18. The erosion distance of [16] between type $\mathcal{B}$ persistence diagrams of constructible persistence modules $F, G$ is

$$
\mathrm{d}_{E}(F, G)=\inf \left\{\varepsilon \geqslant 0 \mid \nabla_{\Omega_{\varepsilon}} F_{\mathcal{B}} \preceq G_{\mathcal{B}} \text { and } \nabla_{\Omega_{\varepsilon}} G_{\mathcal{B}} \preceq F_{\mathcal{B}}\right\},
$$

where $\Omega$ is the usual superlinear family of $\mathbb{R}, \Omega_{\varepsilon}(a)=a+\varepsilon$.

Once again, using the fact that $F_{\mathcal{B}}$ and $G_{\mathcal{B}}$ are Möbius inversions, these inequalities are equivalent to

$$
\nabla_{\Omega_{\varepsilon}} d F_{\mathcal{B}} \leqslant d G_{\mathcal{B}} \text { and } \nabla_{\Omega_{\varepsilon}} d G_{\mathcal{B}} \leqslant d F_{\mathcal{B}},
$$

where the inequalities are pointwise inequalities of functions, i.e. $\nabla_{\Omega_{\varepsilon}} d F_{\mathcal{B}} \leqslant d G_{\mathcal{B}} \Longleftrightarrow$ $\nabla_{\Omega_{\varepsilon}} d F_{\mathcal{B}}(\mathbf{x}) \leqslant d G_{\mathcal{B}}(\mathbf{x})$ for all $\mathbf{x} \in \operatorname{Dgm}_{\mathbb{R}}$. 
This way of getting an erosion distance between persistence modules does not generalize to arbitrary preordered sets $\mathbf{P}$ since we need the $\delta$ in the definition of $d F_{\mathcal{B}}$. Fortunately, forgetting the $\delta$ in the definition turns out to give the same distance as the next proposition and corollary show.

Proposition 3.19. Let $F, G: \mathbb{R} \rightarrow \mathbf{C}$ be constructible persistence modules and $\varepsilon \in$ $[0, \infty)$. Define

$$
\mathcal{F}: \operatorname{Dgm}_{\mathbb{R}} \rightarrow \mathbf{C},(a, b) \mapsto \operatorname{im} F(a<b),
$$

and

$$
\mathcal{G}: \operatorname{Dgm}_{\mathbb{R}} \rightarrow \mathbf{C},(a, b) \mapsto \operatorname{im} G(a<b) .
$$

Now

$$
\nabla_{\Omega_{\varepsilon}} \mathcal{F} \leqslant \mathcal{G} \Longleftrightarrow \nabla_{\Omega_{\varepsilon}} d F_{\mathcal{B}} \leqslant d G_{\mathcal{B}} \Longleftrightarrow \nabla_{\Omega_{\varepsilon}} F_{\mathcal{B}} \preceq G_{\mathcal{B}}
$$

Proof. The right-hand equivalence follows directly from the definition of the rightmost inequality and by the definition of the Möbius inversion. Let $(a, b) \in \operatorname{Dgm}_{\mathbb{R}}$ and we first assume that $\nabla_{\Omega_{\varepsilon}} \mathcal{F} \leqslant \mathcal{G}$. Now, by the definition of $d F_{\mathcal{B}}$ and $d G_{\mathcal{B}}$ there exists $\delta>0$ such that

$$
\nabla_{\Omega_{\varepsilon}} d F_{\mathcal{B}}(a, b)=[\operatorname{im} F(a-\varepsilon<b+\varepsilon-\delta)]
$$

and

$$
d G_{\mathcal{B}}(a, b)=[\operatorname{im} G(a<b-\delta)] .
$$

Hence

$$
\begin{aligned}
\nabla_{\Omega_{\varepsilon}} d F_{\mathcal{B}}(a, b) \leqslant d G_{\mathcal{B}}(a, b) & \Longleftrightarrow[\operatorname{im} F(a-\varepsilon<b+\varepsilon-\delta)] \leqslant[\operatorname{im} G(a<b-\delta)] \\
& \Longleftrightarrow \operatorname{im} F(a-\varepsilon<b+\varepsilon-\delta) \leqslant \operatorname{im} G(a<b-\delta) \\
& \Longleftrightarrow \nabla_{\Omega_{\varepsilon}} \mathcal{F}(a, b-\delta) \leqslant \mathcal{G}(a, b-\delta) .
\end{aligned}
$$

The last inequality holds by assumption, so $\nabla_{\Omega_{\varepsilon}} d F_{\mathcal{B}} \leqslant d G_{\mathcal{B}}$.

Now, we assume that $\nabla_{\Omega_{\varepsilon}} d F_{\mathcal{B}} \leqslant d G_{\mathcal{B}}$ and let $(a, b) \in \operatorname{Dgm}_{\mathbb{R}}$. Since $F$ and $G$ are constructible, there exists a small enough $\delta>0$ such that for all $0<\delta^{\prime} \leqslant \delta$

$$
F\left(b+\varepsilon<b+\varepsilon+\delta^{\prime}\right): F(b+\varepsilon) \cong F\left(b+\varepsilon+\delta^{\prime}\right)
$$

and

$$
G\left(b<b+\delta^{\prime}\right): G(b) \cong G\left(b+\delta^{\prime}\right),
$$

i.e. the morphisms are isomorphisms. Hence

$$
\nabla_{\Omega_{\varepsilon}} d F_{\mathcal{B}}(a, b+\delta)=[\operatorname{im} F(a-\varepsilon<b+\varepsilon)]
$$

and

$$
d G_{\mathcal{B}}(a, b+\delta)=[\operatorname{im} G(a<b)] .
$$

Now

$$
\begin{aligned}
\nabla_{\Omega_{\varepsilon}} \mathcal{F}(a, b) \leqslant \mathcal{G}(a, b) & \Longleftrightarrow \operatorname{im} F(a-\varepsilon<b+\varepsilon) \leqslant \operatorname{im} G(a<b) \\
& \Longleftrightarrow[\operatorname{im} F(a-\varepsilon<b+\varepsilon)] \leqslant[\operatorname{im} G(a<b)] \\
& \Longleftrightarrow \nabla_{\Omega_{\varepsilon}} d F_{\mathcal{B}}(a, b+\delta) \leqslant d G_{\mathcal{B}}(a, b+\delta) .
\end{aligned}
$$

Again, the last inequality holds by assumption, so $\nabla_{\Omega_{\varepsilon}} \mathcal{F} \leqslant \mathcal{G}$. 
Corollary 3.20. Let $F$ and $G$ be constructible persistence modules. Then

$$
\mathrm{d}_{E}^{\Omega}(\mathcal{F}, \mathcal{G})=\mathrm{d}_{E}\left(F_{\mathcal{B}}, G_{\mathcal{B}}\right) .
$$

\section{Minimal preorders and the natural pseudo-distance}

In this section we show how the distance $\mathrm{d}_{T}$ of $[\mathbf{1 1}]$ is obtained as a special case of the erosion distance. The distance $\mathrm{d}_{T}$ is an extended pseudo-metric for continuous functions $\varphi: X \rightarrow \mathbb{R}^{n}$ for some fixed $n \in \mathbb{Z}_{+}$and any topological space $X$, or, more generally, for persistence modules $\mathbb{R}^{n} \rightarrow \mathbf{A b}$. We also show that the recipe for getting a preorder of $\mathbf{A b}$ that is used in $[\mathbf{1 1}]$ can be used in an arbitrary Abelian category $\mathbf{C}$, and that it gives the minimal preorder of $\mathbf{C}$ that respects mono- and epimorphisms.

Throughout this section, we take $\Omega:[0, \infty) \rightarrow \operatorname{Trans}_{\mathbb{R}^{n}}, \Omega_{\varepsilon}(\mathbf{a})=\mathbf{a}+\varepsilon$, where $\varepsilon=$ $(\varepsilon, \ldots, \varepsilon)$.

\subsection{Minimal preorders}

Before looking at the relationship between our general erosion distance and the distance $\mathrm{d}_{T}$, we start with some definitions and propositions to help us declutter the definition of $\mathrm{d}_{T}$ and understand the preorder of $\mathbf{A b}$ that is implicitly defined in [11].

Definition 4.1. We define a preorder for $\mathbf{A b}$ by setting for every $A, B \in \mathbf{A b}$

$$
A \leqslant B \Longleftrightarrow \text { there exists a subgroup } B^{\prime} \subseteq B \text { and an epimorphism } B^{\prime} \rightarrow A \text {. }
$$

In other words, $A \leqslant B$, if and only if $A$ is a subquotient of $B$. This preorder is called the subquotient preorder.

Proposition 4.2. The subquotient preorder is a preorder for $\mathbf{A b .}$

Proof. Reflexivity is trivial. Let $A \leqslant B \leqslant C$, i.e. there exist subgroups $B^{\prime} \subseteq B$ and $C^{\prime} \subseteq C$ such that $B^{\prime} \rightarrow A$ and $f: C^{\prime} \rightarrow B$. We define $C^{\prime \prime}=f^{-1}\left(B^{\prime}\right)$. Now, clearly $C^{\prime \prime} \rightarrow A$, so $A \leqslant C$.

The subquotient preorder clearly respects mono- and epimorphisms. We can define the subquotient preorder in the same way for any category of modules over a ring, and it turns out to be minimal among all preorders that respect mono- and epimorphisms. In a general Abelian category, even if the relation does not define a preorder, its transitive closure is the minimal preorder.

Theorem 4.3. Let $\mathbf{C}$ be an Abelian category. Define a relation $R$ on $\mathbf{C}$ by setting for all $a, b \in \mathbf{C}$

$$
a R b \Longleftrightarrow \text { there exists } b^{\prime} \in \mathbf{C} \text { such that } a \nleftarrow b^{\prime} \hookrightarrow b .
$$

Let $\leqslant$ be the transitive closure of $R$. Then $\leqslant$ is minimal among all preorders of $\mathbf{C}$ that respect mono- and epimorphisms, i.e. if $\preceq$ is another preorder that respects monoand epimorphisms, then for all $a, b \in \mathbf{C}$

$$
a \leqslant b \Rightarrow a \preceq b .
$$

In addition, let $\mathbf{P}$ be a preordered set and fix a superlinear family $\Omega$ (resp. a sublinear projection $\omega)$ of $\mathbf{P}$. Then, the erosion distance with respect to $\leqslant$ and $\Omega$ (resp. $\omega$ ) 
is maximal among all erosion distances of functions $\operatorname{Dgm}_{\mathbf{P}} \rightarrow \mathbf{C}$ with respect to $\Omega$ (resp. $\omega)$.

Proof. Let $a, b \in \mathbf{C}$ such that $a R b$, i.e. there exists $b^{\prime} \in \mathbf{C}$ such that

$$
a \leftarrow b^{\prime} \hookrightarrow b .
$$

Since $\preceq$ respects mono- and epimorphisms, $a \preceq b^{\prime} \preceq b$, hence $a \preceq b$. If $a \leqslant b$, then we have a chain of relations $a R b_{1}, b_{1} R b_{2}, \ldots, b_{m} R b$, which gives us $a \preceq b_{1} \preceq \cdots \preceq b$.

Since the subquotient preorder clearly respects mono- and epimorphisms, we get a stable extended pseudo-metric $\mathrm{d}_{E}^{\Omega}$ for persistence modules $F, G: \mathbb{R}^{n} \rightarrow \mathbf{A b}$ by setting

$$
\mathrm{d}_{E}^{\Omega}(F, G)=\mathrm{d}_{E}^{\Omega}(\mathcal{F}, \mathcal{G})
$$

as in Definition 3.5, where $\mathcal{F}$ and $\mathcal{G}$ are the rank invariants of $F$ and $G$, respectively.

Definition 4.4. We denote

$$
\operatorname{Dgm}_{\mathbb{R}^{n}}^{\prime}:=\left\{(\mathbf{a}, \mathbf{b}) \in \mathbb{R}^{n} \mid a_{i}<b_{i} \text { for each } i=1, \ldots, n\right\} \subseteq \operatorname{Dgm}_{\mathbb{R}^{n}} .
$$

Let $F$ and $G$ be persistence modules and $\mathcal{F}$ and $\mathcal{G}$ be their rank invariants, respectively. We define the distance $\mathrm{d}_{T}$ between $F$ and $G$ to be

$$
\mathrm{d}_{T}(F, G)=\inf \left\{\varepsilon \in[0, \infty)\left|\left(\nabla_{\Omega_{\varepsilon}} \mathcal{F}\right)\right|_{\operatorname{Dgm}_{\mathbb{R}^{n}}^{\prime}} \leqslant\left.\mathcal{G}\right|_{\operatorname{Dgm}_{\mathbb{R}^{n}}^{\prime}} \text { and }\left.\left(\nabla_{\Omega_{\varepsilon}} \mathcal{G}\right)\right|_{\operatorname{Dgm}_{\mathbb{R}^{n}}^{\prime}} \leqslant\left.\mathcal{F}\right|_{\left.\operatorname{Dgm}_{\mathbb{R}^{n}}^{\prime}\right\}}\right. \text {. }
$$

We now have two distances for persistence modules $F, G: \mathbb{R}^{n} \rightarrow \mathbf{A b}: \mathrm{d}_{E}^{\Omega}(F, G)$ and $\mathrm{d}_{T}(F, G)$. The only difference between $\mathrm{d}_{T}$ and $\mathrm{d}_{E}^{\Omega}$ is that the inequalities considered in the definition of $\mathrm{d}_{E}^{\Omega}$ are of functions defined over $\operatorname{Dgm}_{\mathbb{R}^{n}}$ while in the definition of $\mathrm{d}_{T}$ the inequalities are of the same functions restricted to $\operatorname{Dgm}_{\mathbb{R}^{n}}^{\prime} \subseteq \operatorname{Dgm}_{\mathbb{R}^{n}}$. Hence,

$$
\mathrm{d}_{T}(F, G) \leqslant \mathrm{d}_{E}^{\Omega}(F, G) .
$$

The converse inequality actually holds as well, as the next proposition implies.

Proposition 4.5. Let $f, g: \operatorname{Dgm}_{\mathbb{R}^{n}} \rightarrow \mathbf{G}$ be decreasing functions and $\varepsilon>0$ such that $\left.\nabla_{\Omega_{\varepsilon}} f\right|_{\operatorname{Dgm}_{\mathbb{R}^{n}}^{\prime}} \leqslant\left. g\right|_{\operatorname{Dgm}_{\mathbb{R}^{n}}^{\prime}}$. Then $\nabla_{\Omega_{\varepsilon^{\prime}}} f \leqslant g$ for all $\varepsilon^{\prime}>\varepsilon$.

Proof. Let $\varepsilon^{\prime}>\varepsilon$ and take any $(\mathbf{a}, \mathbf{b}) \in \operatorname{Dgm}_{\mathbb{R}^{n}}$. Note that since $\varepsilon^{\prime}-\varepsilon>0$, we have

$$
\left(\mathbf{a}-\left(\varepsilon^{\prime}-\varepsilon\right), \mathbf{b}+\left(\varepsilon^{\prime}-\varepsilon\right)\right) \in \operatorname{Dgm}_{\mathbb{R}^{n}}^{\prime} .
$$

Now, using the inequality $\left.\nabla_{\Omega_{\varepsilon}} f\right|_{\operatorname{Dgm}_{\mathbb{R}^{n}}^{\prime}} \leqslant\left. g\right|_{\operatorname{Dgm}_{\mathbb{R}^{n}}^{\prime}}$ and the fact that $g$ is decreasing,

$$
\begin{aligned}
\nabla_{\Omega_{\varepsilon^{\prime}}} f(\mathbf{a}, \mathbf{b}) & =f\left(\mathbf{a}-\boldsymbol{\varepsilon}^{\prime}, \mathbf{b}+\boldsymbol{\varepsilon}^{\prime}\right) \\
& =\nabla_{\Omega_{\varepsilon}} f\left(\mathbf{a}-\left(\boldsymbol{\varepsilon}^{\prime}-\boldsymbol{\varepsilon}\right), \mathbf{b}+\left(\boldsymbol{\varepsilon}^{\prime}-\boldsymbol{\varepsilon}\right)\right) \\
& \leqslant g\left(\mathbf{a}-\left(\boldsymbol{\varepsilon}^{\prime}-\boldsymbol{\varepsilon}\right), \mathbf{b}+\left(\boldsymbol{\varepsilon}^{\prime}-\boldsymbol{\varepsilon}\right)\right) \\
& \leqslant g(\mathbf{a}, \mathbf{b}) .
\end{aligned}
$$

Hence $\nabla_{\Omega_{\varepsilon^{\prime}}} f \leqslant g$.

As noted before the previous proposition, we get the following proposition.

Proposition 4.6. For all persistence modules $F, G: \mathbb{R}^{n} \rightarrow \mathbf{A b}$

$$
\mathrm{d}_{T}(F, G)=\mathrm{d}_{E}^{\Omega}(F, G) .
$$




\subsection{The natural pseudo-distance}

One of the central results of $[\mathbf{1 1}]$ is Theorem 2.9 that states that $\mathrm{d}_{T}$ gives a lower bound for the natural pseudo-distance. The natural pseudo-distance is a dissimilarity measure between size pairs. It measures how close we can get two functions corresponding to two size pairs, with respect to the $L^{\infty}$-distance, by changing the base space of one of the functions to the base space of the other function by a homeomorphism.

Definition 4.7. A size pair $(X, \varphi)$ consists of a topological space $X$ and a continuous function $\varphi: X \rightarrow \mathbb{R}^{n}$.

Definition 4.8. The natural pseudo-distance between two size pairs $(X, \varphi),(Y, \psi)$ is

$$
\mathrm{d}_{N P}(\varphi, \psi)=\inf _{h \in \operatorname{Homeo}(X, Y)}\|\varphi-\psi \circ h\|_{\infty},
$$

where $\operatorname{Homeo}(X, Y)$ is the set of homeomorphisms from $X$ to $Y, \mathbb{R}^{n}$ is equipped with the max-norm $\|\mathbf{x}\|=\max _{i=1, \ldots, n}\left|x_{i}\right|$, and $\|f\|_{\infty}=\sup _{x \in X}\|f(x)\|$ is the sup-norm.

For more on the natural pseudo-distance, see, e.g., $[\mathbf{8}, \mathbf{9}, \mathbf{1 0}]$, and to see how the natural pseudo-distance can be interpreted as an interleaving distance, see $[\mathbf{7}$, Theorem 3.12].

Theorem 4.9 (Lower bound for the natural pseudo-distance). Let $(X, \varphi)$ and $(Y, \psi)$ be size pairs. Let $H$ : Top $\rightarrow \mathbf{C}$ be a functor, where $\mathbf{C}$ is an Abelian category equipped with a preorder that respects mono- and epimorphisms. The functions $\varphi$ and $\psi$ induce functors $H^{X, \varphi}, H^{Y, \psi}: \mathbb{R}^{n} \rightarrow \mathbf{C}$,

$$
H^{X, \varphi}(\mathbf{a})=H(X\langle\varphi \leqslant \mathbf{a}\rangle), H^{Y, \psi}(\mathbf{a})=H(Y\langle\psi \leqslant \mathbf{a}\rangle),
$$

where $X\langle\varphi \leqslant \mathbf{a}\rangle=\{x \in X \mid \varphi(x) \leqslant \mathbf{a}\}$ and $Y\langle\psi \leqslant \mathbf{a}\rangle=\{y \in Y \mid \psi(y) \leqslant \mathbf{a}\}$ for all $\mathbf{a} \in \mathbb{R}^{n}$. Now

$$
\mathrm{d}_{E}^{\Omega}\left(H^{X, \varphi}, H^{Y, \psi}\right) \leqslant \mathrm{d}_{N P}(\varphi, \psi) .
$$

Proof. We denote the rank invariants of $H^{X, \varphi}$ and $H^{Y, \psi}$ by $\mathcal{H}^{X, \varphi}$ and $\mathcal{H}^{Y, \psi}$.

Let $h: X \rightarrow Y$ be a homeomorphism and set $\varepsilon=\|\varphi-\psi \circ h\|_{\infty}$. We first note that $\left\|\varphi \circ h^{-1}-\psi\right\|_{\infty}=\varepsilon$. We need to show that $\nabla_{\Omega_{\varepsilon}} \mathcal{H}^{X, \varphi} \leqslant \mathcal{H}^{Y, \psi}$ and $\nabla_{\Omega_{\varepsilon}} \mathcal{H}^{Y, \psi} \leqslant \mathcal{H}^{X, \varphi}$. We'll show only the former inequality since the latter can be shown in exactly the same way. Let $(\mathbf{a}, \mathbf{b}) \in \operatorname{Dgm}_{\mathbb{R}^{n}}$. Note that $h$ and $h^{-1}$ can be restricted to maps

$$
X\langle\varphi \leqslant \mathbf{a}-\varepsilon\rangle \stackrel{h}{\rightarrow} Y\langle\psi \leqslant \mathbf{a}\rangle
$$

and

$$
Y\langle\psi \leqslant \mathbf{b}\rangle \stackrel{h^{-1}}{\longrightarrow} X\langle\varphi \leqslant \mathbf{b}+\varepsilon\rangle
$$

since

$$
\begin{aligned}
\varphi(x) \leqslant \mathbf{a}-\varepsilon & \Rightarrow \varphi_{i}(x) \leqslant a_{i}-\varepsilon \forall i=1, \ldots, n \\
& \Rightarrow \psi_{i}(h(x))=\psi_{i}(h(x))-\varphi_{i}(x)+\varphi_{i}(x) \leqslant \varepsilon+a_{i}-\varepsilon=a_{i} \forall i \\
& \Rightarrow \psi(h(x)) \leqslant \mathbf{a}
\end{aligned}
$$

and similarly $\psi(x) \leqslant \mathbf{b} \Rightarrow \varphi\left(h^{-1}(x)\right) \leqslant \mathbf{b}+\varepsilon$. Then, note that the composition of 
maps

$$
X\langle\varphi \leqslant \mathbf{a}-\varepsilon\rangle \stackrel{h}{\rightarrow} Y\langle\psi \leqslant \mathbf{a}\rangle \subseteq Y\langle\psi \leqslant \mathbf{b}\rangle \stackrel{h^{-1}}{\longrightarrow} X\langle\varphi \leqslant \mathbf{b}+\varepsilon\rangle
$$

is simply the inclusion

$$
X\langle\varphi \leqslant \mathbf{a}-\varepsilon\rangle \subseteq X\langle\varphi \leqslant \mathbf{b}+\varepsilon\rangle .
$$

Since $H$ is a functor, we get a commutative diagram

$$
\begin{aligned}
& H(X\langle\varphi \leqslant \mathbf{a}-\varepsilon\rangle) \stackrel{H}{\longrightarrow} H(X\langle\varphi \leqslant \mathbf{b}+\varepsilon\rangle) . \\
& \downarrow H(h) \quad H\left(h^{-1}\right) \uparrow \\
& H(Y\langle\psi \leqslant \mathbf{a}\rangle) \longrightarrow H(Y\langle\psi \leqslant \mathbf{b}\rangle)
\end{aligned}
$$

The image of the upper horizontal map is $\nabla_{\Omega_{\varepsilon}} \mathcal{H}^{X, \varphi}(\mathbf{a}, \mathbf{b})=\mathcal{H}^{X, \varphi}(\mathbf{a}-\varepsilon, \mathbf{b}+\boldsymbol{\varepsilon})$ and the image of the lower horizontal map is $\mathcal{H}^{Y, \psi}(\mathbf{a}, \mathbf{b})$. By Lemma 3.4, $\nabla_{\Omega_{\varepsilon}} \mathcal{H}^{X, \varphi}(\mathbf{a}, \mathbf{b}) \leqslant$ $\mathcal{H}^{Y, \psi}(\mathbf{a}, \mathbf{b})$, and further $\nabla_{\Omega_{\varepsilon}} \mathcal{H}^{X, \varphi} \leqslant \mathcal{H}^{Y, \psi}$.

Remark 4.10. Note that the previous proof can be modified to give a proof for the fact that the interleaving distance gives a lower bound for the natural pseudodistance, i.e.

$$
\mathrm{d}_{I}^{\Omega}\left(H^{X, \varphi}, H^{Y, \psi}\right) \leqslant \mathrm{d}_{N P}(\varphi, \psi) .
$$

This is done by noting that the last commutative diagram shows that $H(h)$ and $H\left(h^{-1}\right)$ give an $\Omega_{\varepsilon}$-interleaving between the functors $H^{X, \varphi}$ and $H^{Y, \psi}$. Then, since we already showed that the erosion distance is smaller than the interleaving distance (Theorem 3.11) we get the theorem.

A third way to prove the theorem is to use the fact the persistent sublevel set homology of a size pair remains invariant when we change the base space with a homeomorphism (see, e.g., [12, Appendix A]). This fact, combined with the classical stability theorem of persistent homology, shows that the interleaving distance gives a lower bound for the natural pseudodistance. Then, we can again use Theorem 3.11 to show the previous theorem.

Corollary 4.11 ([11, Theorem 2.9]). Let $(X, \varphi)$ and $(Y, \psi)$ be size pairs and let $H_{k}: \mathbf{T o p} \rightarrow \mathbf{A b}$ be the $k$-th singular homology functor with coefficients in an Abelian group. Then

$$
\mathrm{d}_{T}\left(H_{k}^{X, \varphi}, H_{k}^{Y, \psi}\right)=\mathrm{d}_{E}^{\Omega}\left(H_{k}^{X, \varphi}, H_{k}^{Y, \psi}\right) \leqslant \mathrm{d}_{N P}(\varphi, \psi) .
$$

\section{Adjunction relation}

If we have a superlinear family and a sublinear projection for $\mathbf{P}$, a natural question is, when are the two erosion distances equal. In [2] it was shown that if the family and the projection satisfy the so-called adjunction relation, then the two interleaving distances are equal. The same argument can be applied to show the equality of the two interleaving distances in our case where the set of translations is smaller. In this section we show that the same conclusion holds for the two erosion distances.

In this section $\mathbf{P}$ is a preordered set, $\mathbf{G}$ is a preordered class, and $\mathbf{C}$ is an Abelian category equipped with a preorder that respects mono- and epimorphisms. 
Definition $5.1([2])$. Let $\Omega:[0, \infty) \rightarrow$ Trans $_{\mathbf{P}}$ and $\omega: \operatorname{Trans}_{\mathbf{P}} \rightarrow[0, \infty]$ be a superlinear family and a sublinear projection, respectively. We say that $\omega$ and $\Omega$ satisfy the adjuction relation, if for all $\varepsilon \in[0, \infty)$ and $\Gamma \in$ Trans $_{\mathbf{P}}$

$$
\omega_{\Gamma} \leqslant \varepsilon \Longleftrightarrow \Gamma \leqslant \Omega_{\varepsilon} .
$$

We also say that $\omega$ and $\Omega$ are an adjoint pair. We denote this relation by $\omega \dashv \Omega$.

Example 5.2. For $\mathbf{P}=\mathbb{R}^{n}$ we have the standard superlinear family $\Omega_{\varepsilon}(\mathbf{a})=\mathbf{a}+\varepsilon$, where $\varepsilon=(\varepsilon, \ldots, \varepsilon)$. Let $\omega: \operatorname{Trans}_{\mathbb{R}^{n}} \rightarrow[0, \infty]$ be the sublinear projection

$$
\omega_{\Gamma}=\sup _{\mathbf{a} \in \mathbb{R}^{n}}\|\mathbf{a}-\Gamma \mathbf{a}\|_{\infty} .
$$

Now, for all $\varepsilon \in[0, \infty)$ and $\Gamma \in \operatorname{Trans}_{\mathbb{R}^{n}}$

$$
\begin{aligned}
\omega_{\Gamma} \leqslant \varepsilon & \Longleftrightarrow\|\mathbf{a}-\Gamma \mathbf{a}\|_{\infty} \leqslant \varepsilon \text { for all } \mathbf{a} \in \mathbb{R}^{n} \\
& \Longleftrightarrow \Gamma \mathbf{a} \leqslant \mathbf{a}+\varepsilon \text { for all } \mathbf{a} \in \mathbb{R}^{n} \\
& \Longleftrightarrow \Gamma \leqslant \Omega_{\varepsilon} .
\end{aligned}
$$

Hence, $\omega \dashv \Omega$.

Note that since all the categories in the definition are thin, the relation is almost precisely the adjunction relation of functors, with the only difference being that the domain of $\Omega$ is not equal to the codomain of $\omega$. If $\operatorname{Trans}_{\mathbf{P}}$ has a maximum, i.e. a translation that is larger than every other translation, then we can extend the domain of $\Omega$ to $[0, \infty]$ and we'll get an adjoint pair of functors.

Before showing that the erosion distances of an adjoint pair $\omega \dashv \Omega$ are equal, we'll give a description of how $\Omega$ (resp. $\omega$ ) determines $\omega$ (resp. $\Omega$ ).

Theorem 5.3 ([17, 2.19]). ${ }^{3}$

i) Let $\Omega:[0, \infty) \rightarrow$ Trans $_{\mathbf{P}}$ be a superlinear family. There exists a sublinear projection $\omega: \operatorname{Trans}_{\mathbf{P}} \rightarrow[0, \infty]$ such that $\omega \dashv \Omega$, if and only if for all $\Gamma \in$ Trans $_{\mathbf{P}}$ the set

$$
\left\{\varepsilon \in[0, \infty) \mid \Gamma \leqslant \Omega_{\varepsilon}\right\} \cup\{\infty\}
$$

has a minimum. If $\omega$ exists, then $\omega_{\Gamma}$ is the minimum of the above set for every $\Gamma \in$ Transp.

ii) Let $\omega:$ Trans $_{\mathbf{P}} \rightarrow[0, \infty]$ be a sublinear projection. There exists a superlinear family $\Omega:[0, \infty) \rightarrow$ Trans $_{\mathbf{P}}$ such that $\omega \dashv \Omega$, if and only if for all $\varepsilon \in[0, \infty)$ the set

$$
\left\{\Gamma \in \operatorname{Trans}_{\mathbf{P}} \mid \omega_{\Gamma} \leqslant \varepsilon\right\}
$$

has a maximum. ${ }^{4}$ If $\Omega$ exists, then $\Omega_{\varepsilon}$ is one of the possibly many maximums of the above set for every $\varepsilon \in[0, \infty)$.

\footnotetext{
${ }^{3}$ Note that the same proof shows the theorem in the setting of [2] where the set of translations is larger.

${ }^{4} \mathrm{By}$ a maximum we mean an element of the set that is larger than every other element of the set. Since $\mathbf{P}$ is only preordered, Trans $\mathbf{P}$ is also only preordered, and so the maximums might not be unique.
} 
Proof.

i) We first assume that $\omega$ exists and let $\Gamma \in \operatorname{Trans}_{\mathbf{P}}$. Since

$$
\omega_{\Gamma} \leqslant \varepsilon \Longleftrightarrow \Gamma \leqslant \Omega_{\varepsilon}
$$

for all $\varepsilon \geqslant 0$, we see that if $\omega_{\Gamma}=\infty$, the set consists only of $\infty$, and if $\omega_{\Gamma}<\infty$, then $\Gamma \leqslant \Omega_{\omega_{\Gamma}}$ by setting $\varepsilon=\omega_{\Gamma}$. Hence $\omega_{\Gamma}$ is in the set. If $\varepsilon$ is in the set as well, then $\Gamma \leqslant \Omega_{\varepsilon}$, and further $\omega_{\Gamma} \leqslant \varepsilon$. Hence, $\omega_{\Gamma}$ is a lower bound, and consequently, the minimum.

Now we'll assume that the minimums exist, and we'll show that the assignment

$$
\omega_{\Gamma}=\min \left(\left\{\varepsilon \in[0, \infty) \mid \Gamma \leqslant \Omega_{\varepsilon}\right\} \cup\{\infty\}\right)
$$

defines a sublinear projection such that $\omega \dashv \Omega$. First, since $I \leqslant \Omega_{0}$, we get $\omega_{I}=0$. Let $\Gamma, K \in$ Trans $_{\mathbf{P}}$. If $\Gamma \leqslant \mathrm{K}$, then

$$
\left\{\varepsilon \in[0, \infty) \mid \mathrm{K} \leqslant \Omega_{\varepsilon}\right\} \subseteq\left\{\varepsilon \in[0, \infty) \mid \Gamma \leqslant \Omega_{\varepsilon}\right\},
$$

and hence $\omega_{\Gamma} \leqslant \omega_{\mathrm{K}}$.

If $\omega_{\Gamma}=\infty$ or $\omega_{\mathrm{K}}=\infty$, then clearly $\omega_{\Gamma \mathrm{K}} \leqslant \omega_{\Gamma}+\omega_{\mathrm{K}}$. If $\omega_{\Gamma}, \omega_{\mathrm{K}}<\infty$, then $\Gamma \leqslant$ $\Omega_{\omega_{\Gamma}}$ and $K \leqslant \Omega_{\omega_{K}}$. Since $\Omega$ is a superlinear family,

$$
\Omega_{\omega_{\Gamma}} \Omega_{\omega_{\mathrm{K}}} \leqslant \Omega_{\omega_{\Gamma}+\omega_{\mathrm{K}}},
$$

and further $\Gamma K \leqslant \Omega_{\omega_{\Gamma}+\omega_{K}}$. Hence $\omega_{\Gamma K} \leqslant \omega_{\Gamma}+\omega_{K}$. This shows that $\omega$ is a sublinear projection.

Clearly

$$
\left\{\varepsilon \in[0, \infty) \mid \Gamma \leqslant \Omega_{\varepsilon}\right\} \cup\{\infty\}=\left[\omega_{\Gamma}, \infty\right]
$$

and further

$$
\omega_{\Gamma} \leqslant \varepsilon \Longleftrightarrow \varepsilon \in\left[\omega_{\Gamma}, \infty\right]=\left\{\varepsilon^{\prime} \in[0, \infty) \mid \Gamma \leqslant \Omega_{\varepsilon^{\prime}}\right\} \cup\{\infty\} \Longleftrightarrow \Gamma \leqslant \Omega_{\varepsilon}
$$

for all $\varepsilon \geqslant 0$. Hence $\omega \dashv \Omega$.

ii) This case can be proven using similar arguments so we leave it to the reader.

Proposition 5.4. Let $\Omega$ be a superlinear family and $\omega$ be a sublinear projection such that $\omega \dashv \Omega$.

i) Let $\Gamma, \Gamma^{\prime}, \mathrm{K}, \mathrm{K}^{\prime} \in$ Trans $_{\mathbf{P}}$ such that $\Gamma \leqslant \Gamma^{\prime}$ and $\mathrm{K} \leqslant \mathrm{K}^{\prime}$. Then, for all decreasing maps $f: \operatorname{Dgm}_{\mathbf{P}} \rightarrow \mathbf{G}$

$$
\nabla_{\Gamma^{\prime}, \mathrm{K}^{\prime}} f \leqslant \nabla_{\Gamma, \mathrm{K}} f
$$

ii) For all decreasing maps $f, g: \operatorname{Dgm}_{\mathbf{P}} \rightarrow \mathbf{G}$

$$
\mathrm{d}_{E}^{\Omega}(f, g)=\mathrm{d}_{E}^{\omega}(f, g) \text {. }
$$

Proof.

i) Let $(a, b) \in \operatorname{Dgm}_{\mathbf{P}}$. Now

$$
\Gamma^{\prime-1} a \leqslant \Gamma^{-1} a \text { and } \mathrm{K} b \leqslant \mathrm{~K}^{\prime} b,
$$

so $\left(\Gamma^{-1} a, \mathrm{~K} b\right) \leqslant\left(\Gamma^{\prime-1} a, \mathrm{~K}^{\prime} b\right)$. Since $f$ is decreasing, we get

$$
\nabla_{\Gamma^{\prime}, \mathrm{K}^{\prime}} f(a, b)=f\left(\Gamma^{\prime-1} a, \mathrm{~K}^{\prime} b\right) \leqslant f\left(\Gamma^{-1} a, \mathrm{~K} b\right)=\nabla_{\Gamma, \mathrm{K}} f(a, b) .
$$

Hence $\nabla_{\Gamma^{\prime}, \mathrm{K}^{\prime}} f \leqslant \nabla_{\Gamma, \mathrm{K}} f$. 
ii) Let $\varepsilon \in[0, \infty)$. We first assume that $\nabla_{\Omega_{\varepsilon}} f \leqslant g$ and $\nabla_{\Omega_{\varepsilon}} g \leqslant f$. By setting $\Gamma=\Omega_{\varepsilon}$ in the defining equivalence of the adjunction relation, we see that $\omega_{\Omega_{\varepsilon}} \leqslant \varepsilon$. Hence we can choose $\Gamma=\mathrm{K}=\Omega_{\varepsilon}$ and now $\omega_{\Gamma}, \omega_{\mathrm{K}} \leqslant \varepsilon$ and $\nabla_{\Gamma, \mathrm{K}} f \leqslant g$ and $\nabla_{\mathrm{K}, \Gamma} g \leqslant f$. This shows that

$$
\mathrm{d}_{E}^{\Omega}(f, g) \geqslant \mathrm{d}_{E}^{\omega}(f, g) .
$$

Next, we assume that there exists $\Gamma, \mathrm{K} \in \operatorname{Trans}_{\mathbf{P}}$ such that $\omega_{\Gamma}, \omega_{\mathrm{K}} \leqslant \varepsilon$ and $\nabla_{\Gamma, \mathrm{K}} f \leqslant g, \nabla_{\mathrm{K}, \Gamma} g \leqslant f$. Since $\omega_{\Gamma}, \omega_{\mathrm{K}} \leqslant \varepsilon$, the adjunction relation implies that $\Gamma, \mathrm{K} \leqslant \Omega_{\varepsilon}$. Hence, by part i) of this theorem, $\nabla_{\Omega_{\varepsilon}} f \leqslant \nabla_{\Gamma, \mathrm{K}} f$ and $\nabla_{\Omega_{\varepsilon}} g \leqslant \nabla_{\mathrm{K}, \Gamma} g$, and further

$$
\nabla_{\Omega_{\varepsilon}} f \leqslant g \text { and } \nabla_{\Omega_{\varepsilon}} g \leqslant f
$$

This shows that

$$
\mathrm{d}_{E}^{\Omega}(f, g) \leqslant \mathrm{d}_{E}^{\omega}(f, g) .
$$

Corollary 5.5. For all persistence modules $F, G: \mathbf{P} \rightarrow \mathbf{C}$

$$
\mathrm{d}_{E}^{\Omega}(F, G)=\mathrm{d}_{E}^{\omega}(F, G) .
$$

\section{References}

[1] H. Bjerkevik and M. Botnan, Computational complexity of the interleaving distance, 34th International Symposium on Computational Geometry (SoCG $2018) 99$ (2018), 13:1-13:15.

[2] P. Bubenik, V. de Silva, and J. Scott, Metrics for generalized persistence modules, Found. Comput. Math. 15 (2015), 1501-1531.

[3] G. Carlsson, Topology and data, Bull. Amer. Math. Soc. 46 (2009), 255-308.

[4] G. Carlsson and A. Zomorodian, The theory of multidimensional persistence, Discrete Comput. Geom. 42 (2009), 71-93.

[5] F. Chazal, V. de Silva, M. Glisse, and S. Oudot, The structure and stability of persistence modules, SpringerBriefs in Mathematics, Springer International Publishing, 2016.

[6] D. Cohen-Steiner, H. Edelsbrunner, and J. Harer, Stability of persistence diagrams, Discrete Comput. Geom. 37 (2007), 103-120.

[7] V. de Silva, E. Munch, and A. Stefanou, Theory of interleavings on categories with a flow, Theory Appl. Categ. 33 (2018), 583-607.

[8] P. Donatini and P. Frosini, Natural pseudodistances between closed manifolds, Forum Math. 16 (2004), 695-715.

[9] P. Donatini and P. Frosini, Natural pseudodistances between closed surfaces, J. Eur. Math. Soc. (JEMS) 9 (2007), 331-353.

[10] P. Donatini and P. Frosini, Natural pseudo-distances between closed curves, Forum Math. 21 (2009), 981-999.

[11] P. Frosini, Stable comparison of multidimensional persistent homology groups with torsion, Acta Appl. Math. 124 (2013), 43-54.

[12] P. Frosini and G. Jabłoński, Combining persistent homology and invariance groups for shape comparison, Discrete Comput. Geom. 55 (2016), 373-409. 
[13] R. Ghrist, Barcodes: the persistent topology of data, Bull. Amer. Math. Soc. 45 (2008), 61-75.

[14] M. Lesnick, The theory of the interleaving distance on multidimensional persistence modules, Found. Comput. Math. 15 (2015), 613-650.

[15] S. Mac Lane, Categories for the working mathematician, 2nd edition, Graduate Texts in Mathematics, Springer-Verlag, New York, 1998.

[16] A. Patel, Generalized persistence diagrams, J. Appl. Comput. Topol. 1 (2018), 397-419.

[17] V. Puuska, Yleiset persistenssimodulit ja lomitusmetriikat, Master's thesis, University of Tampere, 2016.

[18] A. Zomorodian and G. Carlsson, Computing persistent homology, Discrete Comput. Geom. 33 (2005), 249-274.

Ville Puuska puuskaville@gmail.com

Faculty of Information Technology and Communication Sciences, Tampere University, FI-33014 Tampere University, Finland 